\title{
Magnetization reversal and configurational anisotropy of dense permalloy dot arrays
}

\author{
Xiaobin Zhu ${ }^{\text {a) }}$ and P. Grütter \\ Center for the Physics of Materials, Department of Physics, McGill University, 3600 University Street, \\ Montréal Québec H3A 2T8, Canada \\ V. Metlushko \\ Department of Electrical and Computer Engineering, University of Illinois at Chicago, \\ Chicago, Illinois 60607-0024 \\ B. llic \\ School of Applied and Engineering Physics, Cornell University, Ithaca, New York 14853
}

(Received 12 February 2002; accepted for publication 1 May 2002)

\begin{abstract}
Electron beam patterned permalloy circular dots of $700 \mathrm{~nm}$ diameter with small separations were studied by magnetic force microscopy (MFM) in the presence of an in situ magnetic field. Images in the demagnetized state show that the dot is in a vortex state with a vortex core (singularity) in the center. Local hysteresis loops, measured by cantilever frequency shift in an external field, indicate that the magnetization reversal of individual disks is a vortex nucleation and annihilation process. By carefully doing MFM, nucleation and annihilation fields without MFM tip stray field distortions are obtained. Configurational anisotropy originated from magnetostatic coupling is found through hysteresis loops. (C) 2002 American Institute of Physics. [DOI: 10.1063/1.1489720]
\end{abstract}

The magnetic structure and magnetization reversal of small magnets have been widely studied due to fundamental research interest and application potential in high density storage media and magnetoresistive random access memory devices. As the particle size becomes smaller, it is energetically unfavorable to form domain structures. Elliptical particles or elongated particles with a high aspect ratio can form single domain structures, while particles with a circular shape will form a vortex state. ${ }^{1,2}$ Recently, the vortex structure, magnetization behavior, and interdot coupling have been studied by Lorentz microscopy, ${ }^{1}$ magnetic force microscopy (MFM), ${ }^{3}$ magneto optical Kerr effects, ${ }^{4,5}$ and alternating gradient magnetometery. ${ }^{6}$ In this letter, we will use MFM with in situ magnetic fields to study the vortex structures and its magnetization reversal. A local hysteresis loop technique is developed to study the reversal mechanism. The configurational anisotropy originating from interdot coupling is directly measured through hysteresis curves obtained by MFM as well as imaging.

Standard electron-beam lithography and lift-off techniques were used to pattern circular permalloy dots with a diameter of $700 \mathrm{~nm}$ and thickness of $25 \mathrm{~nm}$ on a square rectangular lattice with a lattice constant of $800 \mathrm{~nm}$.

The magnetic structures and magnetization reversal were studied by a custom built vacuum MFM. The experiments were performed in constant height mode to reduce the destructive MFM tip-sample interactions. ${ }^{7}$ Silicon cantilevers with spring constants of 1 and $0.08 \mathrm{~N} / \mathrm{m}$, sputter coated with CoPtCr with thickness of $15 \mathrm{~nm}\left(T_{15}\right), 30 \mathrm{~nm}\left(T_{30}\right)$, and 50 $\mathrm{nm}\left(T_{50}\right)$ were used as magnetic probes. The experiments were performed in a vacuum of $1 \times 10^{-5}$ Torr. A digital

\footnotetext{
a) Author to whom correspondence should be addressed; electronic mail: xbzhu@physics.mcgill.ca
}

phase locked loop from NanoSurf to increase sensitivity was used to decode the cantilever frequency shift. ${ }^{8}$ The typical tip-sample separations were about 50-80 nm, unless otherwise indicated.

The ac demagnetized state of permalloy disks have small stray fields, which lead to very low magnetic contrast MFM images. High-resolution images show that the disk forms a vortex state as shown in Figs. 1(a) and 1(b). The disk shows very weak contrast with a bright spot in the center. This is a flux core, a singularity of the vortex state in the permalloy dot, which has been directly observed only recently. ${ }^{1,3}$ Figures 1(c) and 1(d) are the images when an external field is applied diagonally along the image area. As expected, the flux core moves closer to the edge perpendicularly to the field direction. This result is consistent with experiments
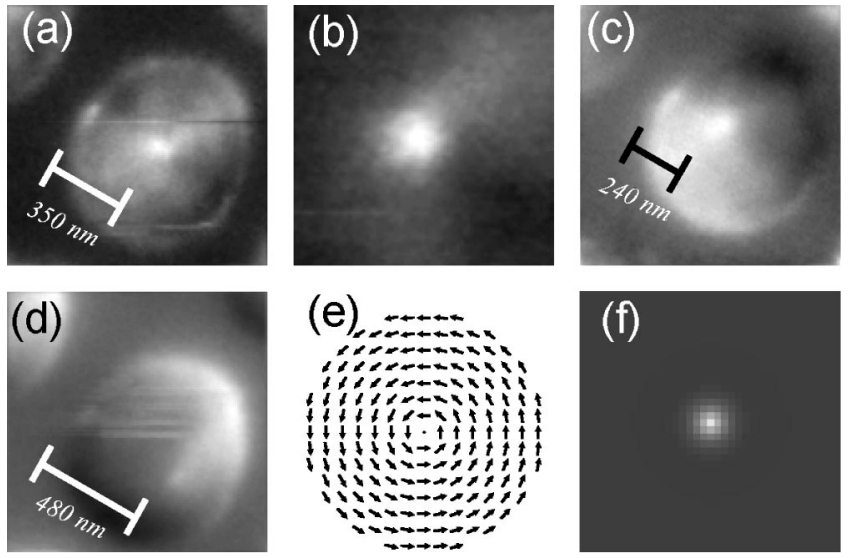

FIG. 1. (a) Vortex structure of permalloy disk; (b) zoom in of a vortex core with $140 \mathrm{~nm}$ scan size; (c) image at a field of $23 \mathrm{Oe}$; (d) image at a field of $-55 \mathrm{Oe}$; (e) simulated moment distribution of a permalloy disk with the same size as the experiment; and (f) gray scale shows the moments of out of plane component in a $140 \mathrm{~nm}$ area of (e). Tip: $T_{30}$. 


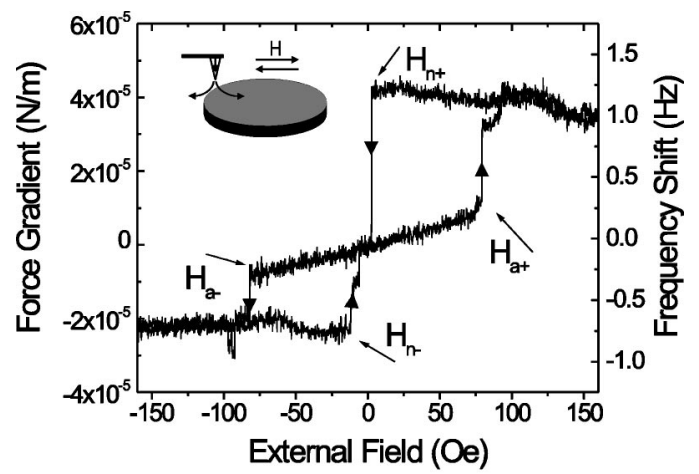

FIG. 2. Force gradient between the MFM tip and sample as a function of external field, while the tip is located at a fixed position $30 \mathrm{~nm}$ above the disk, as shown in the inset. Cantilever: $T_{15}, k=1 \mathrm{~N} / \mathrm{m}$.

done by Lorentz microscopy, ${ }^{1}$ micromagnetic simulation, ${ }^{9}$ and theoretical analysis. ${ }^{10}$ The simulated results, as shown in Figs. 1(e) and 1(f), indicate that the lowest energy state is a vortex state with most of the moments rotating along the disk radius. However, the center part (the vortex core), with a size of about $10 \mathrm{~nm}$, points normal to the sample plane. Because it is small in size, the flux core is hardly observable at large tip-sample separation. The observed MFM contrasts with external field is also consistent with modeling. However, a detailed comparison will be discussed elsewhere.

A distinct characteristic of magnetic disks, which has been confirmed by measuring the hysteresis loop of arrays of dots or through the study of individual disks, ${ }^{1,3-5}$ is that the magnetization reversal has two transitions: the nucleation and annihilation of the magnetic vortex. Here, we develop a local hysteresis technique to characterize the two transitions of a single dot. The local hysteresis loop can be obtained by monitoring the cantilever frequency shift as a function of the external magnetic field, while the tip is located at a fixed position above the disk, as shown in the inset of Fig. 2. The cantilever frequency shift is then converted to the force gradient between magnetic probe and the disk, $F^{\prime}$. This force gradient is approximately proportional to the sample moment. A typical plot is shown in Fig. 2. When examining each single curve from positive (negative) saturation to negative (positive) saturation, we found that two distinct jumps are clearly visible. One is associated with the nucleation process, while the other is associated with the annihilation of a vortex. As the field decreases from positive saturation (single domain state) the force gradient initially remains at a "constant" value. When the field reaches the nucleation field $H_{n}$, the force gradient decreases abruptly (the formation of a vortex is confirmed by imaging). As the field decreases to a negative value, the vortex core moves toward the edge of the disk [Figs. 1(c) and 1(d)], and the force gradient decreases. When the field reaches a critical value $H_{a}$, the force gradient decreases abruptly again and reaches a static value (the vortex is expelled). When the field is increased from negative saturation to positive saturation, similar phenomena can be observed.

Close examination of the hysteresis curve reveals that it is not symmetric. The absolute value of the force gradient is not symmetric, which is due to the $z$ component of the MFM tip stray field locally reversibly rotating the moments of the dots. The switching fields, however, are not symmetric ei- $\quad$ By following this procedure, we can obtain accurate
Downloaded 11 Jul 2011 to 131.193.154.219. Redistribution subject to AIP license or copyright; see http://apl.aip.org/about/rights_and_permissions

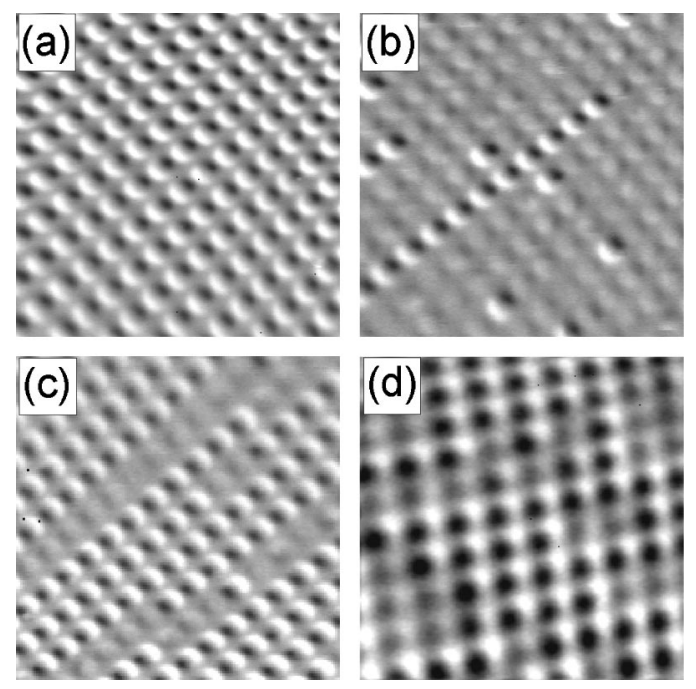

FIG. 3. A series of images after applying different external fields. (a) Field ramped to $126 \mathrm{Oe}$, and imaging at $60 \mathrm{Oe}$; (b) field ramped to $23 \mathrm{Oe}$, and image at $60 \mathrm{Oe}$; (c) field ramped to $-20 \mathrm{Oe}$ after saturation at $-200 \mathrm{Oe}$, and imaged at $-60 \mathrm{Oe}$, and (d) field ramped to -40 Oe after saturation at -200 Oe, and imaged at -70 Oe. Tip: $T_{50}$.

ther. The difference comes from the in-plane component of the tip stray field contributing to the magnetization reversal. Assume that the average in-plane component of tip stray field on the particle is $H_{\text {eff }}$, and the nucleation field without tip is $H_{n}$. We observe $H_{n}=\left(H_{n+}+H_{n-}\right) / 2 \approx 10$ Oe for the disk. The average contribution of the tip $H_{\text {eff }}=\left(H_{n+}\right.$ $\left.-H_{n-}\right) / 2$ is less than 10 Oe for $T_{15}$ and 25 Oe for $T_{30}$ with a tip-sample separation of about $30 \mathrm{~nm}$. Not only can the local hysteresis curve be used to characterize the magnetization behavior of small disks, but it is also possible to use this technique to characterize both in-plane and out of plane components of the tip stray field.

It is challenging to obtain the disk switching field from images ${ }^{11}$ since the tip stray field can induce irreversible distortions. To obtain the switching field of dots, one can assume that the MFM tip contributes a constant field offset. ${ }^{11}$ However, many effects such as the unknown tip in-plane and out-of-plane component, and the potential change of the tip stray field in the external field lead to major uncertainties. To obtain an accurate switching field, one needs to perform the MFM experiments at a field close to $H_{p}=\left(H_{n}+H_{a}\right) / 2$ after the external field is ramped to a designated value $H$. The disk will maintain its previous state at $H_{p}$. Suppose that at $H$ the disk is reversed to a single domain state. When the field is decreased to $H_{p}$, the nucleation of a vortex will not yet happen. Alternatively, suppose that at $H$ the disk is reversed to a vortex state. When the field is increased to $H_{p}$, annihilation will not yet happen. The prerequisite to reliable and accurate determination of a switching field is that $H_{p}$ combined with the tip stray field cannot induce reversal. This can be actually solved by using low moment tips and operating in the constant height mode. ${ }^{7}$ Figure 3 shows a series of images, where the field was applied in the 100 or 110 directions, as shown in the inset Fig. 4. No tip stray field induced reversal can be observed. A weak contrast from the low moment state comes from the distorted vortex as the imaging is not taken at remanence.

By following this procedure, we can obtain accurate e or copyright; see http://apl.aip.org/about/rights_and_permissions 


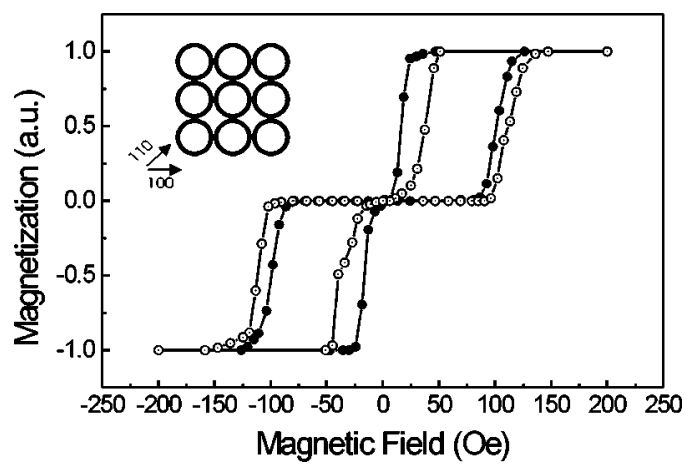

FIG. 4. Hysteresis curves along 100 (solid dots) and 110 (open dots) direction. Tip: $T_{50}$.

switching fields of the disks without tip stray field artifacts. The "hysteresis curve" (switching probability) can be obtained by characterizing ensembles of disks, as shown in Fig. $4 .^{12} \mathrm{~A}$ two step transition can clearly be seen. Figure 4 shows that both the nucleation and annihilation fields depend on the direction of the external field. Table I list the average switching field and its variation. We found that if the field is applied in the 100 direction of the array, a smaller annihilation and nucleation field is observed. The switching fields difference between 100 direction and 110 direction cannot be explained by dipole interaction for uniformly magnetized dots, which only yield a uniaxial anisotropy contribution. The observed anisotropy can be explained by nonuniform magnetization induced anisotropy, configurational anisotropy.,13 Model calculation by Guslienko ${ }^{13}$ shows that the magnetostatic energy is anisotropic with a minima along the 100 or 010 direction with fourfold anisotropy. The anisotropy comes from high order multipole coupling, quadrupolar interaction being dominant. For magnetic disks without an external field, the stray field of each individual disk is very small, and the

TABLE I. Switching field and its width in oersteds.

\begin{tabular}{lcllc}
\hline \hline Field & $\left\langle H_{n}\right\rangle$ & $\delta H_{n}$ & $\left\langle H_{a}\right\rangle$ & $\delta H_{a}$ \\
\hline$\langle 100\rangle$ & 14 & 5 & 104 & 9 \\
$\langle 110\rangle$ & 32 & 9.3 & 115.5 & 11.5 \\
\hline \hline
\end{tabular}

interdot dipolar and higher order multipolar coupling is negligible. For annihilation of a vortex, as the magnetic field is applied, the vortex state is deformed by shifting the vortex core out of center, and the magnetization in the disk is nonuniform. For nucleation of a vortex, as the magnetic field decreases from saturation, the nonuniform shape of the magnetization configuration can be formed before the nucleation of a vortex. Such nonuniformity will then lead to the observed anisotropy. The magnetostatic coupling is expected to be weak for the 110 direction as is directly confirmed by MFM images [Figs. 3(c) and 3(d)]. For the 100 direction, the nucleation appears to be correlated with nucleated chains [Fig. 3(c)]. However, in the 110 direction, the correlation is much weaker [Fig. 3(d)]. This correlation is stronger in nucleating vortices than in annihilating vortices, and leads to smaller switching field variations in the 100 direction than that in the 110 direction.

This work at McGill was supported by grants from NSERC of Canada and FCAR of Québec. This work at UIC was supported by the U.S. NSF Grant No. ECS-0202780 (V.M.).

${ }^{1}$ M. Schneider, H. Hoffman, and J. Zweck, Appl. Phys. Lett. 77, 2909 (2000).

${ }^{2}$ R. P. Cowburn, D. K. Koltsov, A. O. Adeye, and M. E. Welland, Phys. Rev. Lett. 83, 1042 (1999).

${ }^{3}$ T. Shinjo, T. Okuno, R. Hassdorf, K. Shigeto, and T. Ono, Science 289, 930 (2000).

${ }^{4}$ R. P. Cowburn, A. O. Adeyeye, and M. E. Welland, Phys. Rev. Lett. 81, 5414 (1998).

${ }^{5}$ V. Novosad, K. Yu. Guslienko, H. Shima, Y. Otani, S. G. Kim, K. Fukamichi, N. Kikuchi, O. Kitakami, and Y. Shimada, Phys. Rev. B 65, 060402(R) (2002).

${ }^{6}$ M. Schneider and H. Hoffmann, J. Appl. Phys. 86, 4539 (1999).

${ }^{7}$ X. Zhu, P. Grütter, V. Metluskho, and B. Ilic, J. Appl. Phys. 91, 7340 (2002).

${ }^{8}$ Nanosurf AG, Liestal, CH. www.nanosurf.com.

${ }^{9}$ The simulation uses the publicly available three-dimensional CODE from NIST, http://math.nist.gov/oommf. We used a unit cell of $4 \mathrm{~nm} \times 4 \mathrm{~nm}$ $\times 5 \mathrm{~nm}$, an exchange constant of $1.0 \times 10^{-11} \mathrm{~J} / \mathrm{m}$, and the damping constant of 0.5 .

${ }^{10}$ K. Yu. Guslienko, V. Novosad, Y. Otani, H. Shima, and K. Fukamichi, Appl. Phys. Lett. 78, 3848 (2001).

${ }^{11}$ A. Fernandez and C. J. Cerjan, J. Appl. Phys. 87, 1395 (2000).

${ }^{12}$ We assign \pm 1 to the single domain state, and 0 to the vortex state. The hysteresis curve is obtained by averaging over 120 individual disks.

${ }^{13}$ K. Yu. Guslienko, Phys. Lett. A 278, 293 (2001). 